\title{
Measurement of collision-induced shift and broadening of the ultraviolet transitions of $\mathrm{OH}$
}

\author{
B. Shirinzadeh \\ Physics Department, University of Michigan, Ann Arbor, Michigan 48109 \\ D. M. Bakalyar ${ }^{a)}$ \\ Department of Physics, Oakland University, Rochester, Michigan 48063 \\ Charles C. Wang ${ }^{\text {) }}$ \\ Research Staff, Ford Motor Company, Dearborn, Michigan 48121
}

(Received 19 October 1984; accepted 30 November 1984)

\begin{abstract}
Using a cw tunable laser, absorption measurements were made to determine for the first time the shift and broadening of the ultraviolet transitions of $\mathrm{OH}$ induced by collisions with molecular nitrogen and oxygen. Similar measurements were also made for collisions with air and inert gases $\mathrm{He}, \mathrm{Ne}, \mathrm{Ar}$, and $\mathrm{Kr}$. Both the shift and broadening due to these collisions were found to depend linearly upon pressures in the range from 50 Torr up to $1 \mathrm{~atm}$. The homogeneous linewidth for the $P_{1}(2)$ transition of $\mathrm{OH}$ in ambient air is determined to be $(0.20 \pm 0.005) \mathrm{cm}^{-1}$. Results with $\mathrm{Ar}$ and $\mathrm{Kr}$ show contributions not accounted for by the polarizability of the colliding species.
\end{abstract}

With the advent of $\mathrm{cw}$ tunable, single-frequency dye lasers, it has become possible to perform absorption measurements ${ }^{1}$ with much improved sensitivity and detectability. This paper describes measurements of collisioninduced shift and broadening of the ultraviolet transitions of the hydroxyl $(\mathrm{OH})$ radical. The collision-induced broadening is of some importance, since it is involved in the deduction of $\mathrm{OH}$ concentrations from fluorescence measurements ${ }^{2}$ in ambient air. Previous measurements ${ }^{3}$ of collision-induced effects were made with limited sensitivity and were unable to discern any shifts in the transition wavelength. Our results represent the first measurements of both the shift and broadening of the $\mathrm{OH}$ transitions induced by collisions with molecular nitrogen, oxygen, and air. Similar results were also obtained with the inert gases helium, neon, argon, and krypton.

The experimental setup used for our experiment is shown schematically in Fig. 1. As before, ${ }^{1,4}$ the frequency doubled output of a single-frequency dye laser was used as the light source. This output in the UV was tuned across the $P_{1}(2)$ line (or other rotational-electronic lines) in the ${ }^{2} \Pi\left(v^{\prime \prime}=0\right) \rightarrow{ }^{2} \Sigma^{+}\left(v^{\prime}=0\right)$ transitions of $\mathrm{OH}$. It was intensity stabilized with the aid of an electro-optic $(\mathrm{E} / \mathrm{O})$ modulator, and frequency stabilized relative to the absorption signal generated in a water-vapor discharge containing high concentrations $\left(\sim 10^{13}\right.$ molecules $\left./ \mathrm{cm}^{3}\right)$ of $\mathrm{OH}$. In our absorption measurements, the main portion of the beam was split into two parts: one part was detected

a) Present address: Division of Cardiovascular Diseases, William Beaumont Hospital, Royal Oak, Michigan 48072.

b) Author to whom correspondence regarding this manuscript should be addressed. by a photomultiplier tube immediately after the splitting, and the other part made a round trip traversal through the sampled volume, as shown in the schematic, before it was detected by another photomultiplier. The difference between these two detector outputs constituted a spectral signal which was averaged and stored in a signal averager. Comparison of this signal with the reference signal generated in the water-vapor discharge mentioned above was then made using a HP-85 computer.

The harmonic generator (doubler), discharge cell, and frequency and intensity stabilization schemes have been described previously. ${ }^{4}$ The quartz absorption cell used in this experiment measured $69.5 \mathrm{~cm}$ in length and was surrounded by six UV lamps (GE-8G-1490). Buffer gas containing ozone $\left(\sim 10^{13}\right.$ molecules $\left./ \mathrm{cm}^{3}\right)$ was bubbled through distilled water before it passed through the cell with a flow velocity of less than $30 \mathrm{~cm} / \mathrm{sec}$. $\mathrm{OH}$ was generated through the reaction of water vapor with $O\left({ }^{1} D\right)$ produced as a photodissociation product of ozone. The concentration of $\mathrm{OH}$ obtained in this manner was on the order of $10^{12}$ molecules $/ \mathrm{cm}^{3}$. The total pressure in the cell was varied between 50 and 720 Torr, while the ozone concentration was kept roughly constant.

The wavelength of the laser was scanned back and forth across the transition at a rate of about $4 \mathrm{~Hz}$. The data obtained for the two scanning directions were stored and averaged separately; the location of the absorption peak observed in each scanning direction was compared with the corresponding peak observed in the low pressure water-vapor discharge. The results obtained for both scanning directions were averaged to yield the shift in the center frequency of the absorption line. This procedure eliminated systematic errors due to any difference in the electronic phase shift of the amplifiers or difference in the 


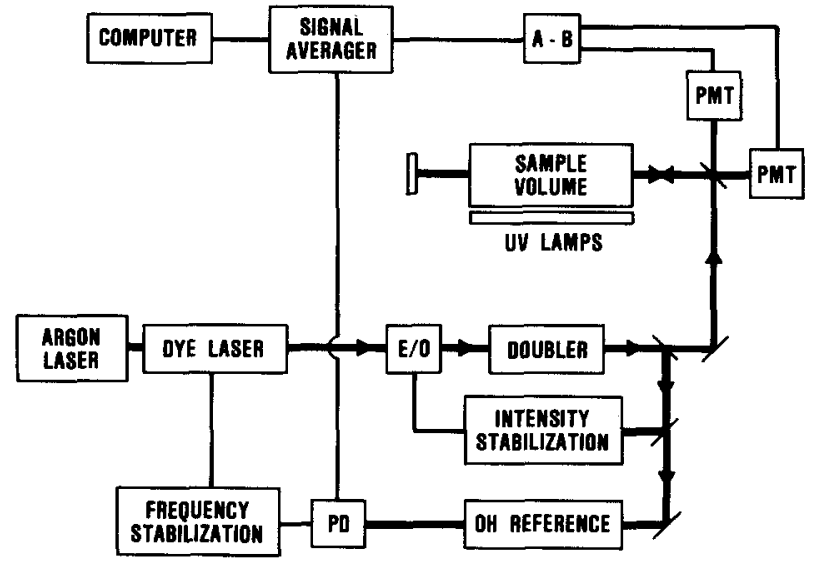

FIG. 1. Schematic of the experimental setup.

time axes of the signal averager channels. The deduced values for the homogeneous broadening were not susceptible to this systematic error, being essentially the same well within the fitting error for the two scanning directions.

It was also found that other effects synchronous with the scanning were associated with the signal base line. These effects were minimized by comparing the absorption signals taken with and without any $\mathrm{OH}$ in the sample and reference cells, and taking the difference between these signals as that due to $\mathrm{OH}$. For the results to be presented in this paper, each absorption profile usually involves ten such on-off cycles, with each cycle consisting of 300 scans. Using these procedures, both the synchronous effects and random fluctuations are reduced to about $10^{-4}$ of the de level.

In an absorption experiment, the intensity $I(\nu)$ of the light beam transmitted through a column of absorbing gas of length $l$ is given by

$$
I(\nu)=I_{0} \exp [-K(\nu) l]
$$

or

$$
K(\nu)=(1 / l) \ln \left[I_{0} / I(\nu)\right],
$$

where $I_{0}$ is the intensity of the incident beam, and $K(\nu)$ is the absorption coefficient of the medium at frequency $\nu$ of the incident beam. In general, $K(\nu)$ assumes a Voigt profile, exhibiting Doppler broadening as well as broadening and shift induced by collisions with other atoms or molecules. The collision-induced shift and broadening, which form the subject of discussion in this paper, was determined through deconvolution of the observed spectral line shape using a procedure of nonlinear least-square fitting (IMSL routine: ZXSSQ). As input to the procedure of least-square fitting, the Doppler width was assumed to be that appropriate for the measured temperature in the cell (typically $23^{\circ} \mathrm{C}$ ), and provisions were also incorporated to allow for possible offsets in the base line. The laser linewidth from a single-mode source was of the order of megahertz, and thus was negligible compared to other sources of broadening.

In the interest of minimizing computer time for the nonlinear least-square fitting, a preliminary fit was performed using an approximate formula for the Voigt profile given by Whiting. ${ }^{5}$ The approximate value thus obtained for the homogeneous linewidth was then used as the initial value for the nonlinear fitting with the exact Voigt function. This latter function is given by

$$
\begin{aligned}
& W(z)=\operatorname{Re}\left[\exp \left(-z^{2}\right) \operatorname{erfc}(-i z)\right], \\
& z=2\left(\nu-\nu_{0}-\Delta \nu_{s}\right) / w_{g}+w_{l} / w_{g},
\end{aligned}
$$

where $w_{g}$ and $w_{l}$ are, respectively, the Gaussian and Lorentzian widths (FWHM), $\nu_{0}$ is the center frequency of the absorption line in the absence of any collision, $\Delta \nu_{s}$ is the shift in this center frequency induced by collision, and $\operatorname{erfc}(-i z)$ is the complemented error function which can be computed readily with the IMSL routine (MERRCZ). In the processing of our data, the results on the shift and broadening deduced according to the Whiting approximation are generally within $2 \%$ of that deduced from the exact Voigt profile.

Figure 2 depicts the absorption signal as a function of detuning under a system pressure of 720 Torr of air. The digitized experimental data were shown as discrete points (although they were obtained as a continuous function of detuning) and the solid curve represents the Voigt profile obtained as a least-square fit to the data. The peak absorption signal is seen to be only about $1.7 \%$, but excellent fit and good signal-to-noise ratio are clearly evident. Generally, the statistical uncertainty associated with the deduced values for the shift and broadening is on the order of $10^{-4} \mathrm{~cm}^{-1}$. The collision-induced shift and broadening deduced from our experimental results are linearly proportional to total pressure within the absorption cell (Fig. 3), thus indicating that the observed effects are the results of two-body collisions. The nonzero intercept for the width reflects the contributions, both resonant and nonresonant, ${ }^{6}$ from water vapor which was kept constant as the air pressure was varied. With the data in Fig. 3, a linear least-square fitting yields a value of $(26.4 \pm 0.7) \times 10^{-5} \mathrm{~cm}^{-1} /$ Torr and $(-2.21 \pm 0.04)$ $\times 10^{-5} \mathrm{~cm}^{-1} /$ Torr for the broadening and shift due to air, respectively. For dry air under the standard condition at sea level, this observed broadening gives a homogeneously broadened linewidth of $(0.20 \pm 0.005) \mathrm{cm}^{-1}$.

We have also measured the shift and broadening

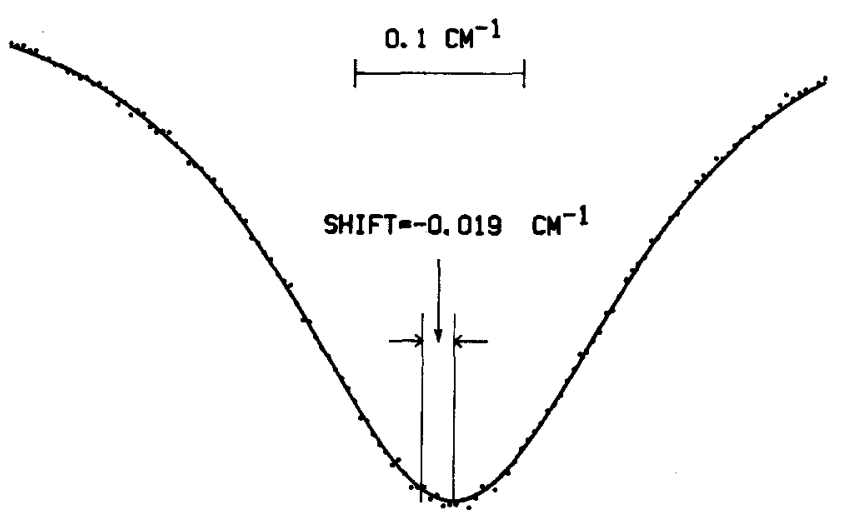

FIG. 2. Absorption signal as a function of detuning near $3068.4 \AA$ the transition frequency of the $P_{1}(2)$ line of $\mathrm{OH}$. (๑): Experimental results; $(-)$ : least-square fit to the experimental points. See text for details. 


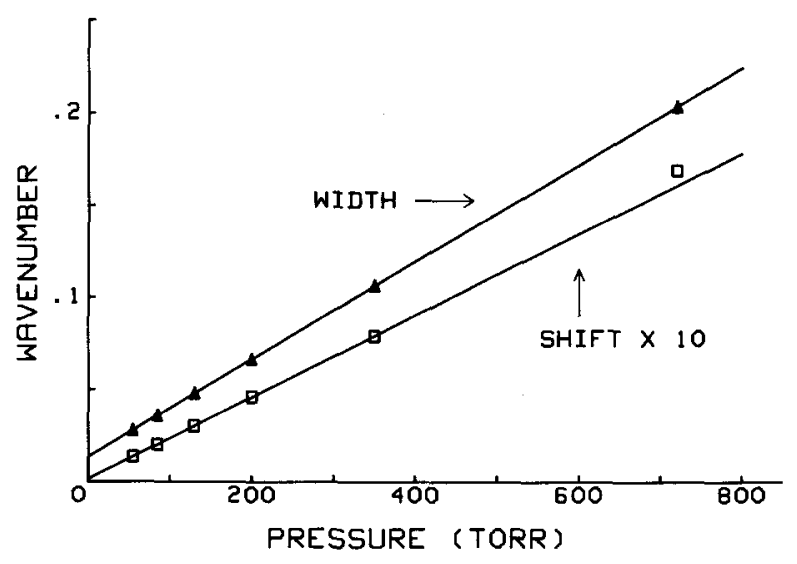

FIG. 3. Pressure dependence of the shift and broadening of the $P_{1}(2)$ line of $\mathrm{OH}$ induced by collision with air molecules.

induced in collisions with nitrogen and oxygen molecules, and the results are summarized in Table $I$. With a composition of $78 \%$ nitrogen and $22 \%$ oxygen for air, one calculates from these results that the broadening and shift coefficients for air should be $(26.4 \pm 0.7) \times 10^{-5}$ $\mathrm{cm}^{-1} /$ Torr and $(-2.24 \pm 0.21) \times 10^{-5} \mathrm{~cm}^{-1} /$ Torr, respectively. These results are in close accord with those deduced from direct measurements of air noted earlier.

In view of recent interests in the study of collisions involving rare gases with diatomic molecules ${ }^{7}$ or atomic species, ${ }^{8}$ we have extended our measurements to include those due to collisions with the rare gases $\mathrm{He}, \mathrm{Ne}, \mathrm{Ar}$, and $\mathrm{Kr}$. The results of these measurements are also tabulated in Table I. Briefly, the observed broadening due to collisions with $\mathrm{Ar}$ and $\mathrm{Kr}$ are about the same as that due to oxygen molecules, but the broadening due to $\mathrm{He}$ and $\mathrm{Ne}$ are smaller by about a factor of 3 . The observed red shift due to the rare gases are in general comparable to those due to molecular oxygen and nitrogen, but otherwise show no systematic trend.

For the sake of discussion, we have also deduced from these measurements the values for the parameters $C_{6}$ and $C_{12}$ of the Lennard-Jones potential. ${ }^{8}$ The deduced values for $C_{12}$ are seen to scale approximately as the 12 th power of the combined radii of the colliding atoms (or
TABLE I. Shift and broadening of the $P_{1}(2)$ transition of $\mathrm{OH}$ deduced from the observed absorption line profile.

\begin{tabular}{lrr}
\hline & \multicolumn{1}{c}{ Width } & Shift \\
\cline { 2 - 3 } Gas & \multicolumn{2}{c}{$10^{-5} \mathrm{~cm}^{-1}$ Torr $^{-1}$} \\
\hline $\mathrm{O}_{2}$ & $18.8 \pm 0.7$ & $-3.36 \pm 0.21$ \\
$\mathrm{~N}_{2}$ & $28.5 \pm 0.7$ & $-1.93 \pm 0.21$ \\
$\mathrm{Air}$ & $26.4 \pm 0.7$ & $-2.21 \pm 0.04$ \\
$\mathrm{He}$ & $7.5 \pm 0.3$ & $-1.31 \pm 0.04$ \\
$\mathrm{Ne}$ & $6.4 \pm 0.7$ & $-1.94 \pm 0.21$ \\
$\mathrm{Ar}$ & $18.5 \pm 0.3$ & $-4.04 \pm 0.04$ \\
$\mathrm{Kr}$ & $18.0 \pm 0.4$ & $-3.46 \pm 0.06$ \\
\hline \hline
\end{tabular}

molecules), ${ }^{9}$ but the deduced values for $C_{6}$ increase much faster than a linear function of the atomic polarizability expected for the Lennard-Jones potential. This indicates, of course, that the Lennard-Jones potential is probably too crude for a quantitative analysis of our data and that other terms in the interaction potential must be taken into account. ${ }^{10}$

It is a pleasure to acknowledge the capable assistance of Thomas Pugsley and Donald Luttermoser during the course of the experiments. This research has been supported in part by National Aeronautics and Space Administration through a grant to Wayne State University, and by the Department of Energy.

' C. C. Wang and B. Shirinzadeh, Phys. Rev. A 28, 1166 (1983); B. Shirinzadeh and C. C. Wang, Appl. Opt. 22, 3265 (1983).

${ }^{2}$ C. C. Wang, L. I. Davis, P. M. Selzer, and R. Munoz, J. Geophys. Res. 86C, 1181 (1981).

${ }^{3}$ R. Engleman, J. Quant. Spectrosc. Radiat. Transfer 9, 391 (1969).

${ }^{4}$ D. M. Bakalyar, J. V. James, and C. C. Wang, Appl. Opt. 22, 2901 (1982).

${ }^{5}$ C. E. Whiting, J. Quant. Spectrosc. Radiat. Transfer 8, 1379 (1969).

${ }^{6}$ C. C. Wang, D. K. Killinger, and C. Huang, Phys. Rev. A 22, 188 (1980).

${ }^{7}$ A. S. Pine, J. Mol. Spectrosc. 82, 435 (1980).

${ }^{8}$ J. E. Thomas, R. A. Forber, L. A. Spinelli, and M. S. Feld, Phys. Rev. Lett. 51, 2194 (1983).

${ }^{9}$ W. R. Hindmarsh, A. D. Petford, and G. Smith, Proc. R. Soc. London Ser. A 297, 296 (1967).

${ }^{10} \mathrm{H}$. Margenau and N. R. Kesiner, Theory of Intermolecular Forces, 2nd ed. (Pergamon, New York, 1971). 